\title{
Zoledronic acid inhibits TSC2-null cell tumor growth via RhoA/YAP signaling pathway in mouse models of lymphangioleiomyomatosis
}

\author{
Dandan Zhao ${ }^{2 \dagger}$, Jing Wu ${ }^{1 \dagger}$, Yinjuan Zhao ${ }^{3}$, Wei Shao ${ }^{1}$, Qi Cheng ${ }^{2}$, Xiaoyan Shao ${ }^{2}$, Xianwen Yuan ${ }^{2,4}$, Juan Ye ${ }^{2,5}$, \\ Jianpu Gao ${ }^{1}$, Meiling Jin ${ }^{6}$, Chaojun $\mathrm{Li}^{2,7}$, Xiaolin Chen ${ }^{9}$, Yue Zhao ${ }^{2,7^{*}}$ and Bin Xue ${ }^{1,2,7,8^{*}}$ (D)
}

\begin{abstract}
Background: This study is to investigate the effects of zoledronic acid (ZA) on TSC2-null cell proliferation and on the tumor progression and recurrence in mouse models of lymphangioleiomyomatosis (LAM).

Methods: Subcutaneous mouse models and LAM mouse models were established. Immunohistochemistry and immunofluorescence were performed to detect the protein expression levels. TUNEL assay was conducted to detect cell apoptosis. Immunoprecipitation was carried out to determine the interaction between proteins.

Results: ZA prevented the growth of TSC2-null cells both in culture and in LAM mouse models. Compared with rapamycin, ZA more effectively promoted the apoptosis of TSC2-null cells. Moreover, combined with the rapamycin, ZA effectively suppressed the tumor recurrence after drug withdrawal and ZA inhibited the activity of GTPase RhoA by decreasing protein geranylgeranylation, resulting in changes of Yap nucleus translocation.
\end{abstract}

Conclusion: ZA promotes cell apoptosis in TSC2-null cells through the RhoA/YAP signaling pathway. ZA may be used for the clinical treatment of LAM.

Keywords: Lymphangioleiomyomatosis, MTOR, Zoledronic acid (ZA), YAP, Autophagy

\section{Background}

Lymphangioleiomyomatosis (LAM) is a destructive cystic lung disease caused by LAM cells, which invade all the lung structures, including the lymphatics, airway wall, blood vessels, and interstitial spaces, therefore limiting the delivery of oxygen to the body. Moreover, LAM is a rare, progressive mesenchymal neoplasm mainly affecting childbearing age women [1-4]. The LAM cases are sometime difficult to diagnose because the disease

\footnotetext{
*Correspondence: zhyue@nju.edu.cn; xuebin@njmu.edu.cn

'Dandan Zhao and Jing Wu contributed equally to this work

${ }^{1}$ Core Laboratory, Sir Run Run Hospital, Nanjing Medical University,

Nanjing 211166, Jiangsu, China

${ }^{2}$ State Key Laboratory of Pharmaceutical Biotechnology, Jiangsu Key

Laboratory of Molecular Medicine and School of Medicine, Nanjing University, Nanjing 210093, Jiangsu, China

Full list of author information is available at the end of the article
}

symptoms are similar to other lung diseases (such as asthma, emphysema, and bronchitis). The high-resolution chest CT scan and VEGF-D blood test could provide accurate diagnosis of LAM $[5,6]$. Each year, 3.3-7.4 million women have been diagnosed, and $0.23-0.31$ million new cases have been confirmed [7]. Up to now, there is still no therapy that could cure LAM, but only the lung transplantation can save the life of LAM patients. In 2000-2006, some studies have found that somatic mutation of tuberous sclerosis complex 2 (TSC2) gene in LAM patients would cause abnormal proliferation of LAM cells $[8,9]$. However, how the TSC2 mutation induces LAM and where these abnormally proliferated LAM cells come from have not yet fully elucidated.

LAM can be sporadic or co-exist with TSC, and all the cases have been found to be associated with function

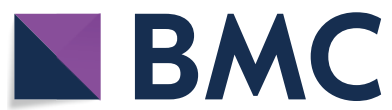

(c) The Author(s) 2020. This article is licensed under a Creative Commons Attribution 4.0 International License, which permits use, sharing, adaptation, distribution and reproduction in any medium or format, as long as you give appropriate credit to the original author(s) and the source, provide a link to the Creative Commons licence, and indicate if changes were made. The images or other third party material in this article are included in the article's Creative Commons licence, unless indicated otherwise in a credit line to the material. If material is not included in the article's Creative Commons licence and your intended use is not permitted by statutory regulation or exceeds the permitted use, you will need to obtain permission directly from the copyright holder. To view a copy of this licence, visit http://creativeco mmons.org/licenses/by/4.0/. The Creative Commons Public Domain Dedication waiver (http://creativecommons.org/publicdomain/ zero/1.0/) applies to the data made available in this article, unless otherwise stated in a credit line to the data. 
loss of TSC2 gene. The TSC2 protein can form a complex with GTPase Rheb to block the activity of mTORC1, thus inhibiting the mTOR pathway [10]. Hence, the loss function mutation of TSC2 gene in LAM patients causes the advanced activation of the mTORC1/mTORC2 pathways. The mTORC1 can phosphorylate several downstream substrates, including the pS6 (Ser235/236) and 4EBP-1. Phosphorylation of the ribosomal protein S6 is increased in the LAM lung tissues, compared with the normal lung tissues [11]. The mTORC2 has been found to be insensitive to rapamycin, which could phosphorylate AKT at the Ser473 site and negatively regulate the AKT/PI3K pathway [12]. Rapamycin and rapalogs are the inhibitors of mTORC1, enhancing the rapid clinical transformation and benefiting many women with LAM $[13,14]$. However, some clinical studies have shown that the lung function would become deteriorated again and relapse would occur after the cessation of rapamycin for 24 months [15, 16]. Moreover, Morris et al. [17] have reported that although mTORC1 is sensitive to rapamycin, mTORC2 is only sensitive to prolonged rapamycin treatment, and there might be drug tolerance. Therefore, multi-drug combination represents an effective method for the treatment of LAM. The mevalonate pathway is an essential metabolic pathway, in which the acetyl-CoA is used to produce sterols and isoprenoids (GGPP and FPP), involved in tumor growth and progression [18]. Bisphosphonate and statins are inhibitors of the mevalonate pathway, with however different target sites [19]. Statins are widely used HMG-CoA reductase inhibitors which affect many cross-talk pathways. Zoledronic acid (ZA) targets on two key enzymes in the mevalonate pathway, i.e., the FPPS and GGPPS, which are essential for the GTPase activity [20]. Several studies have reported that the combination of sivastatin and rapamycin represent effective antitumor intervention in the TSC2-null cells and the LAM mouse model. Moreover, some preliminary clinical researches have also shown that the rapamycin and stains treatments could improve the situations of some TCS and LAM patients [21]. However, long-term administration of statins would induce undesirable side effects, including abnormal lung function [22]. Furthermore, ZA has been found to exert long-term efficacy and safety in the treatment of skeletal metastases with non-small cell lung carcinoma and other solid tumors, without toxicity for patients, such as breast cancer and prostate cancer [23, 24].

In this study, the effects of ZA on TSC2-null cell proliferation and on the tumor progression and recurrence in LAM mouse models, were investigated. Moreover, the underlying mechanisms of ZA inducing effects in TSC2null cells, and the involvement of the RhoA/YAP pathway were also analyzed. Our findings might provide evidence for the application of $\mathrm{ZA}$ in the clinical treatment of LAM.

\section{Methods \\ Cell growth and viability assessment}

TSC2-null MEF cells ( Tsc $2^{-/-} \mathrm{p} 53^{-/-}$) were kindly gift from John Blenis (Weill Cornell Medical College, New York, NY, USA). These cells were cultured in the DMEM medium, supplemented with $10 \%$ FBS and $1 \%$ antibiotics. The viability of TSC2-null cells was detected by the MTT method. Briefly, $5 \times 10^{3}$ cells were seeded onto 96-well plates and treated with indicated concentrations of ZA. After $24 \mathrm{~h}, 50 \mu \mathrm{l} 1 \times$ MTT solution (diluted by dilution buffer, KeyGENBioTECH) was added and incubated for $4 \mathrm{~h}$. Then, $150 \mu \mathrm{l}$ DMSO was added. The absorbance at $550 \mathrm{~nm}$ was measured by the Multiscan Spectrum.

\section{Animals and treatments}

The 6-8-week-old STOCK-Foxn1nu/Nju female nude athymic mice were brought from the Model Animal Research Center of Nanjing University (Nanjing, Jiangsu, China). All animal procedures were carried out according to the guideline of the Animal Care and Use Committee of the Model Animal Research Center of Nanjing University. Mice were housed in a 12-h light/dark cycle, with free access to food and water. Animals did not suffer unnecessarily at any stage of an experiment.

\section{Subcutaneous mouse model establishment}

For the subcutaneous model establishment, female nude mice were subcutaneously injected with $5 \times 10^{6}$ TSC2null cells in right flank. When tumors grew to $3 \mathrm{~mm}$ in diameter, the mice were injected with $\mathrm{ZA}(1 \mathrm{mg} / \mathrm{kg} / 3$ day; dissolved in 30\% PEG400) through the tail vein, for 30 day. For the control group, mice were injected with placebo dissolved in 30\% PEG400 through tail vein. Tumor volumes were calculated according to the following formulation: Tumor volume $=$ Length $\times \mathrm{Width}^{2} \times 0.5$. Animal weight were monitored and recorded.

\section{LAM mouse model establishment}

For the establishment of LAM mouse models, female nude mice were subcutaneously injected with $5 \times 10^{6}$ TSC2-null cells in both flanks, for 30-40 day. When the tumor volume reached $1 \mathrm{~cm}$ in diameter, mice were sacrificed. The tumors were removed and enzymatically digested with Collagenase Type IV and cultured with DMEM medium, supplemented with 10\% FBS and 1\% antibiotics. After 2 day, TSC2-null cells were harvested from the dishes and sifted through mesh, and $1 \times 10^{6}$ cells were injected into the tail vein of female nude mice [25]. 
For both models, mice were injected with rapamycin ( $1 \mathrm{mg} / \mathrm{kg} / 3$ day), ZA $(200 \mu \mathrm{g} / \mathrm{kg} / 2$ day and $1 \mathrm{mg} / \mathrm{kg} / 3$ day, respectively), or combination of ZA ( $1 \mathrm{mg} / \mathrm{kg} / 3$ day) and rapamycin ( $1 \mathrm{mg} / \mathrm{kg} / 3$ day). Rapamycin were injected intraperitoneally and ZA were injected through the tail vein. Animal weights were monitored and recorded. To determine whether the withdrawal of ZA would induce the tumor recurrence, the rapamycin treatment alone and the combination of rapamycin and ZA for 30 day was terminated. After $1 \mathrm{~m}$, the animals were sacrificed to check the tumor recurrence.

\section{Immunohistochemistry detection}

Mouse tissues were fixed in $4 \%$ paraformaldehyde, embedded in paraffin, and cut into $5-\mu \mathrm{m}$ sections. Tissue sections were deparaffinized and hydrated before being incubated in $10 \mathrm{mmol} / \mathrm{l}$ citrate buffer $(\mathrm{pH}=6)$ for $10 \mathrm{~min}$ in a microwave oven. Sections were blocked with goat serum for $1 \mathrm{~h}$, and then incubated with indicated primary antibodies (Additional file 1: Table S1) at $4{ }^{\circ} \mathrm{C}$ overnight. Next, the sections were incubated with biotinylated secondary antibodies, and then immunoreactions were revealed by the DAB chromogen system.

\section{Immunofluorescence}

Cell slides were fixed with $4 \%$ paraformaldehyde, washed with PBS, and then permeabilized with $0.1 \%$ Triton- 100 for $15 \mathrm{~min}$. Next, slides were blocked in goat serum for $1 \mathrm{~h}$, and then incubated with the indicated primary antibodies (Additional file 1: Table S1) at $4{ }^{\circ} \mathrm{C}$ overnight. Alex Fluor 594- and Alex Flour 488-conjugate secondary antibodies were used. After stained with DAPI, immunofluorescence was detected.

\section{TUNEL assay}

Apoptotic cells were detected by using DeadEnd ${ }^{\mathrm{TM}}$ Fluorometric TUNEL System (Promega, Madison, WI, USA), according to the manufacturer's instructions. Immunofluorescence results was analyzed using the Image-Pro Expression system and the ImageJ software.

\section{Immunoblotting analysis}

Cells were lysed in cell lysis buffer (RIPA) with complete protease inhibitor cocktail (Roche, Pleasanton, CA, USA) and centrifuged at $120,000 g$ at $4{ }^{\circ} \mathrm{C}$ for $15 \mathrm{~min}$. The cytosolic fraction in the supernatant was collected. Protein extract was separated by SDS-PAGE, and then electronically transferred onto the PVDF membrane (Roche). After blocking, the membrane was incubated with indicated primary antibodies (Additional file 1: Table S1) at $4{ }^{\circ} \mathrm{C}$ for overnight. After washing, the membrane was incubated with secondary antibody. Subsequently, immunoblotting was performed using the chemiluminescence method (Millipore, Bedford, MA, USA), according to manufacturer's instructions.

\section{Immunoprecipitation}

Membrane and cytoplasmic protein extracts were prepared with the Triton X-114 partition method [26]. These membrane and cytoplasmic proteins were immunoprecipitated using the RhoA protein antibody (Santa Cruz, Santa Cruz, CA, USA), followed by the Western blot analysis.

\section{Statistical analysis}

All data were presented as mean \pm SEM. Comparison between groups was performed using the Student's $t$ test. $P<0.05$ was considered statistically significant.

\section{Results}

ZA exerts antitumor effects in TSC2-null cell subcutaneous tumor model and LAM mouse model

The antitumor potential of ZA on subcutaneous tumor model was first examined. The nude mice were subcutaneously injected with TSC2-null cells, and then treated with ZA ( $1 \mathrm{mg} / \mathrm{kg} / 3$ day; through tail vein injection). The doses of ZA were determined according to the clinical application for patients with bone disorders. To determine the effects of ZA on TSC2-null tumor growth, the tumor size and mouse weights were monitored and recorded three times a week. Our results showed that, after the ZA treatment, no significant changes were observed in the general behavior and mouse weight between the placebo and ZA groups. However, the tumors grew more quickly in the placebo group compared with the ZA treatment group, with significant difference for the time point of 3 weeks (Fig. 1a, b). Because the tumor volumes in the ZA group were decreased, the Ki67 staining was performed for the tumor tissues at 30 day after ZA treatment. Our results showed that the ZA treatment efficiently inhibited the TSC2-null cell proliferation (Fig. 1c).

To further confirm whether $\mathrm{ZA}$ influenced the mTORC1 activity in TSC2-null cells just like rapamycin, the phosphorylation of S6 (p-S6) was checked by immunofluorescence in tumor tissues. Our results showed that the p-S6 expression was weaker in the ZA group, indicating that ZA could decrease the p-S6 expression in the TSC2-null cell tumor (Fig. 1d). Advanced activation of mTOR pathway makes TSC2-null cells have impaired autophagic flux. We wondered whether ZA could induce autophagy in TSC2-null cells. Firstly, we checked the p-S6 expression in vitro to further confirm ZA affect the activity of mTOR pathway. Our results showed that the p-S6 was partly reduced in the high-concentration ZA group (Additional file 2: Fig. S1A). Moreover, the accumulation 
a

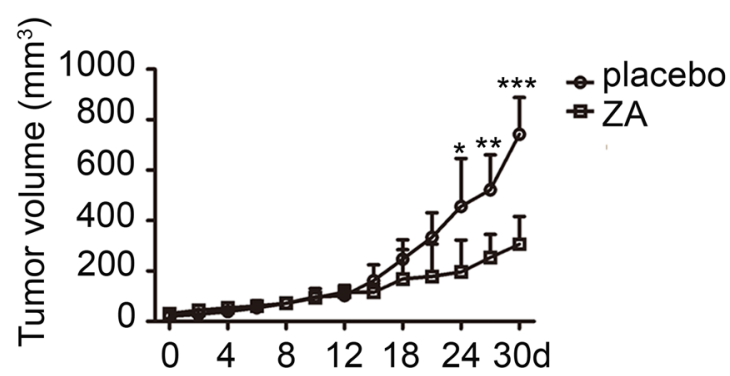

b

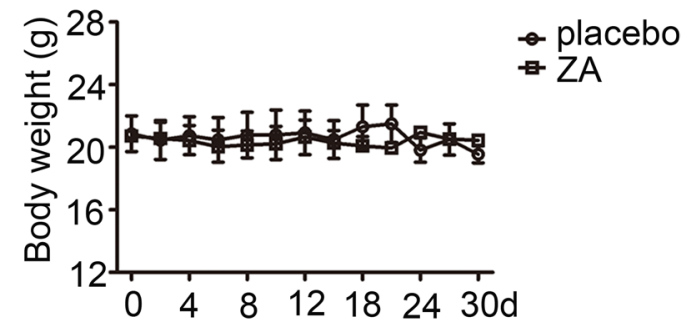

c
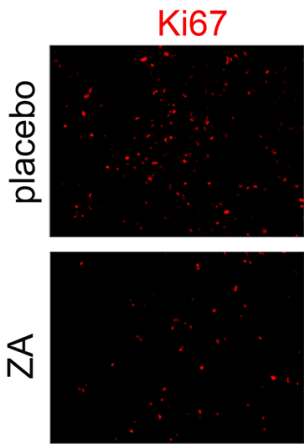

d
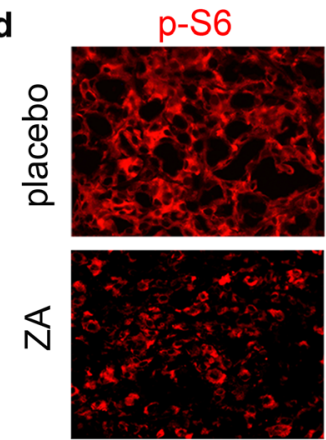

e

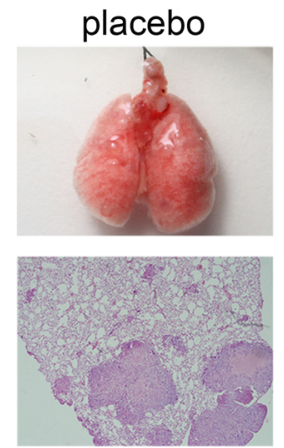

DAPI
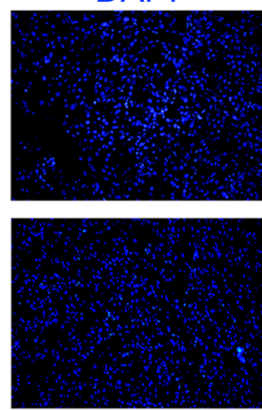

DAPI
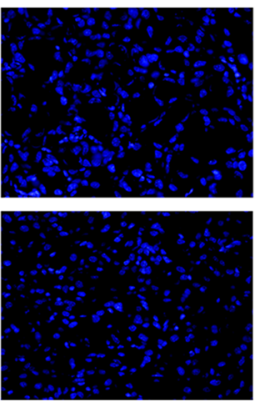

ZA 200

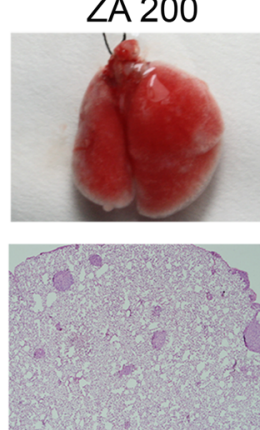

Ki67/DAPI
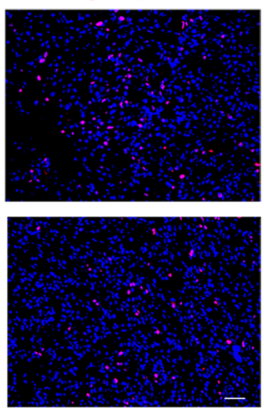

p-S6/DAPI
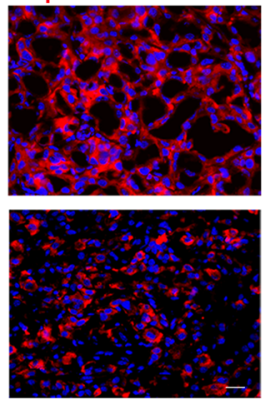

ZA 1000

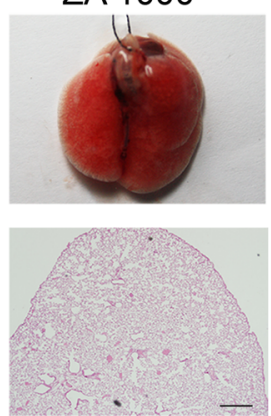

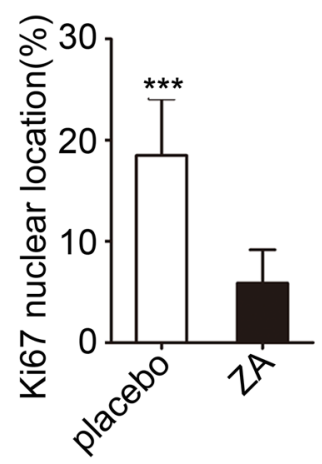
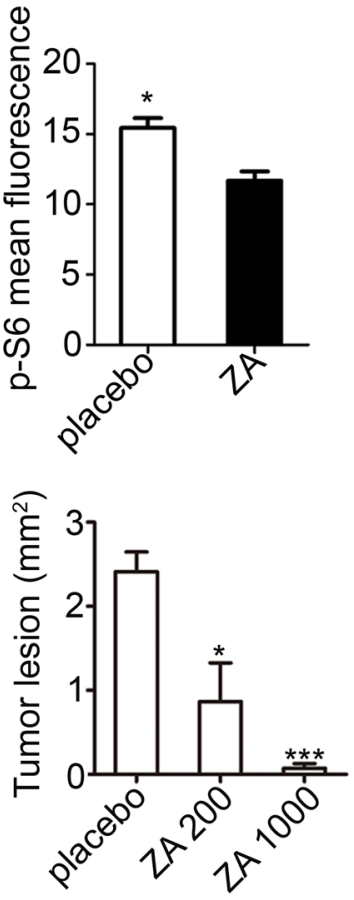

Fig. 1 ZA exerts antitumor activity in TSC2-null cell subcutaneous tumor model and LAM mouse model. a Tumor growth in STOCK-Foxn1nu/Nju female athymic nude mice with TSC2-null cells given by $1 \mathrm{mg} / \mathrm{kg} / 3$ day zoledronic acid (ZA) through the tail vein $(n=6)$. b Body weights of nude mice with TSC2-null cell tumors were subjected to 30-d treatment with placebo and ZA, respectively $(n=6)$. c Immunofluorescence of Ki67 in the subcutaneous mice model. Scar bar, $100 \mu m(n=6)$. d Tumor lesion sections stained for p-S6 (ser 234/235) $(n=6)$. Scar bar, $50 \mu m$. e H\&E staining of lung lesions of placebo- and ZA-injected mice after tail vein injection of TSC2-null cells for 30 day $(n=6)$. Scar bar, $500 \mu m$. The experiment was performed for three times. All data were presented as mean \pm SEM. Compared with the placebo, ${ }^{* *} P<0.01,{ }^{* * *} P<0.001$ 
of LC3-II was observed in the ZA treatment group (Additional file 2: Fig. S1B). This phenomenon was also confirmed by the immunofluorescence staining of LC3 and LAMP1 (Additional file 2: Fig. S1C). In addition, the GGPP treatment rescued the excessive autophagy in ZA-treated TSC2-null cells (Additional file 2: Fig. S1D). Together, these findings suggest that ZA could cause TSC2-null cell autophagy by inhibiting GGPP.

In order to simulate the clinical features of LAM patients, the mouse model of LAM was re-built with TSC2-null cells. The lung morphology observation showed that TSC2-null cell lesions in mice tended to accumulate around the veins and arteries [there were no other tumor lesions in other organs (Additional file 3: Fig.S2B)]. The mTORC1 activity marker p-S6 was also checked, and the H\&E staining was performed. Our results showed significantly increased space in the alveolar airspace (Additional file 3: Fig. S2C) and higher p-S6 expression level (Additional file 3: Fig. S2A) compared with the normal lung structure. Next, tumor growth and the alveolar destruction in LAM mouse model were assessed. We observed that inhibition of tumor growth depended on drug concentrations of ZA (Fig. 1e).

\section{ZA induces apoptosis of TSC2-null cell via inhibiting GGPP}

To further determinate the antitumor effects of ZA in vitro, TSC2-null cells were treated with indicated concentrations of ZA. We also found that ZA inhibited the TSC2-null cell proliferation, in a dose-dependent manner (Fig. 2a). Moreover, comparing with rapamycin, ZA inhibited cell proliferation and induced apoptosis more effectively (Fig. 2b). These results were also confirmed by the immunofluorescence staining of Ki67 (Fig. 2c) and the DeadEnd ${ }^{\mathrm{TM}}$ Fluorometric TUNEL System analysis (Fig. 2d). To assess which subtract of ZA affects the proliferation of TSC2-null cells, FPP and GGPP were added into the culture medium, respectively. Our results showed that the apoptosis could be rescued by the FPP and GGPP both (Fig. 2e). However, FPP could produce GGPP by geranylgeranyl pyrophosphate synthase (GGPPS) in the cells. Treatment of GGPP alone (without FPP) could rescue the inhibitory effect of $\mathrm{ZA}$, suggesting that $\mathrm{ZA}$ exerted anticancer effects because of inhibition of GGPP.

\section{ZA induces apoptosis of TSC2-null cells by inactivating RhoA/YAP pathway}

Some study showed that Yap is essential for TSC2null cell. To specifically validate Yap function could be affected by ZA, the expression levels of Yap was checked after treating with different concentrations of ZA. Our results showed that the Yap expression level was steadily decreased in a dose-dependent manner with ZA treatment (Fig. 3a). Next, we over-expressed YAP in the
TSC2-null cells and showed that cleaved-caspase-3 and PARP was sharply attenuated after YAP over-expression (Fig. 3b), indicating that the apoptosis of TSC2-null cells induced by ZA was blunted by the YAP over-expression. These results were further confirmed by detection of $\mathrm{TUNEL}^{+}$apoptotic cells (Fig. 3c). RhoA plays an important role in Yap function. We next checked RhoA expression in the cell membrane and cytoplasm by immunoprecipitation and we found that the expression of RhoA in the cell membrane was reduced (Fig. 3d). Yap expression was also examined in the subcutaneous tumor model by immunofluorescence and showed that the expression of Yap was sharply attenuated in the ZA group (Fig. 3e). Moreover, GGPP can rescue the Yap nuclear translocation in vitro (Fig. 3f). Collectively, these date demonstrate that ZA induces apoptosis of TSC2null cells by blocking GGPP leading to inhibition of Yap.

\section{ZA induces autophagy leading to Yap protein degradation in TSC2-null cells}

Autophagy usually causes proteins degradation. To check whether the decreased Yap protein expression was caused by autophagy, the mRNA expression of Yap was detected. Our result showed that there was no difference in the Yap mRNA level after the ZA treatment (Additional file 4: Fig. S3C). These results suggest that the Yap protein down-regulation may be due to protein degradation, which may be caused by autophagy, but not decrease of YAP mRNA. Moreover, the ZA treatment could induce an excessive level of autophagy (Additional file 2: Fig. S1D). Furthermore, when the TSC2-null cells were treated with the autophagy inhibitor CQ, the Yap expression and cell proliferation would be partly rescued (Additional file 4: Fig. S3A, B). These results suggest that autophagy induced by ZA in the TSC2-null cells mainly showed the dark-side effects. Based on these findings, we hypothesize that ZA might induce excessive autophagy in the TSC2-null cells, leading to the Yap degradation and eventually causing the cellular apoptosis.

\section{Combination of ZA and rapamycin inhibits tumor recurrence after drug withdrawal}

Resistance of rapamycin has been described in some tumor cells and clinical cases. In this study, whether the combination of ZA and rapamycin inhibited the tumor growth in LAM mouse model was assessed. The MTT analysis showed that combination of $\mathrm{ZA}$ and rapamycin inhibited the growth of TSC2-null cells more effectively (Fig. 4a). Importantly, the combination of ZA and rapamycin showed significant synergistic effects compared with the rapamycin treatment alone, which stopped the tumor recurrence after drug withdrawal, with minor lung lesions (Fig. 4b, c). These results indicate that the 

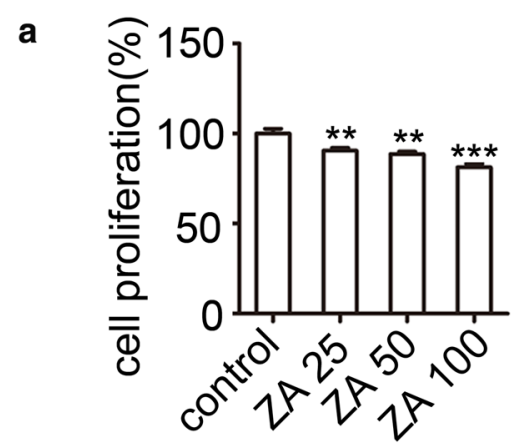

c
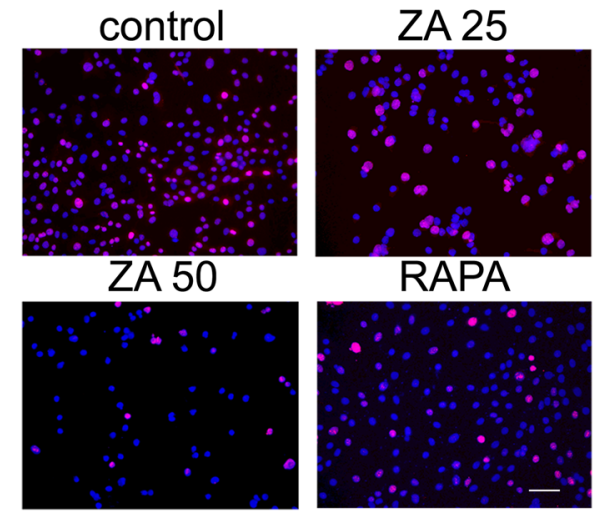

d
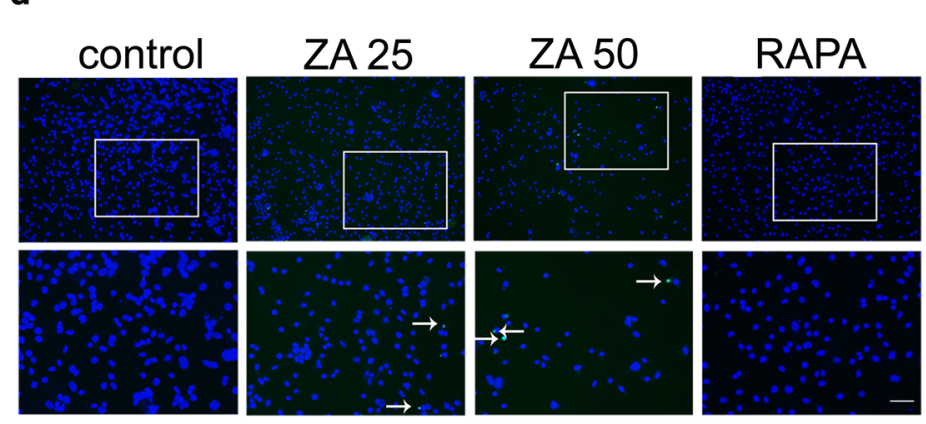

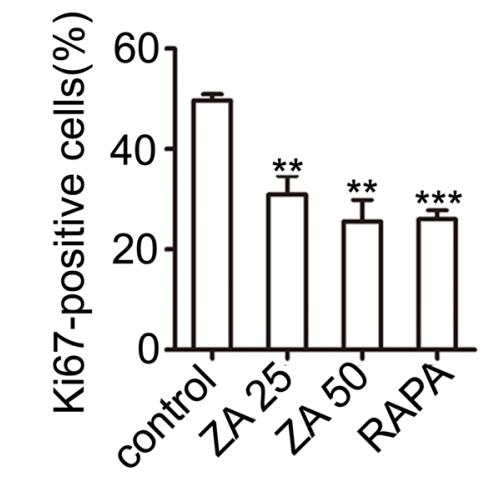

b

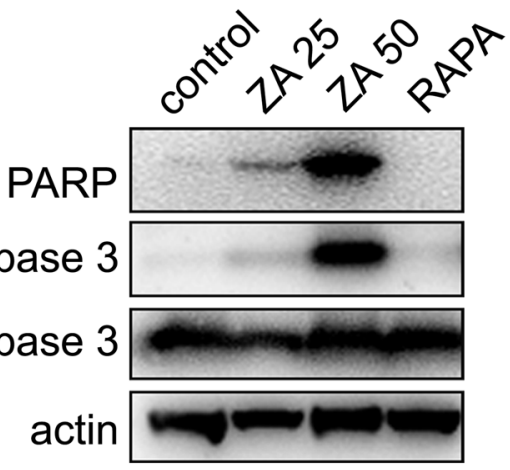

caspase 3

actin

cleaved-caspase 3

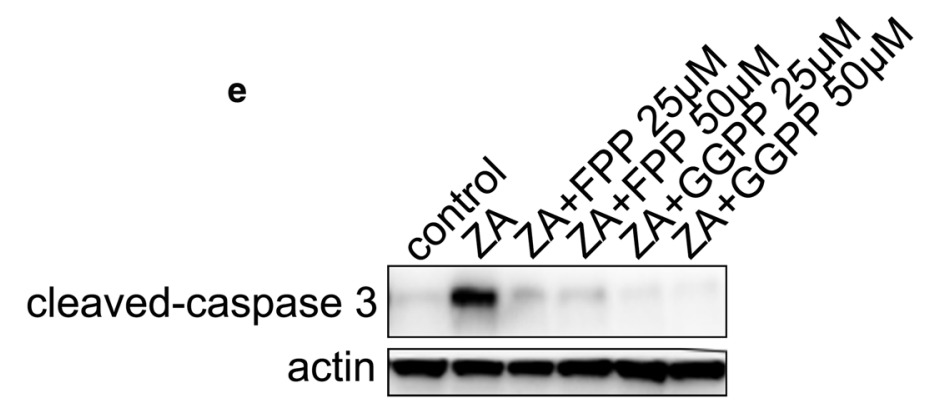

Fig. 2 ZA induces TSC2-null cell apoptosis by GGPP. a MTT assay of TSC2-null cells treated with control, as well as 25, 50, and 100 $\mu$ M ZA. b Western blot analysis after treatment with ZA (25 and $50 \mu \mathrm{M}$, respectively; and $20 \mathrm{nM}$ RAPA for 24 h) in TSC2-null cells. c Immunostaining of Ki67 after treatment with ZA (25 and $50 \mu \mathrm{M}$; and $20 \mathrm{nM}$ RAPA for $24 \mathrm{~h}$ ). Immunofluorescence staining was performed and analyzed in independent 3 experiments. $\mathbf{d}$ Apoptosis of TSC2-null cells treated with ZA assayed using the DeadEnd ${ }^{\text {TM }}$ Fluorometric TUNEL System. The green dots represented apoptotic cells, and DAPI (blue) indicated cell nuclei. Scar bar, 100 and $200 \mu \mathrm{m}$, respectively. e Immunoblotting analysis of apoptosis marker (cleaved-caspase3) after treatment with ZA alone, the combination of ZA and FPP, and the combination of ZA and GGPP, respectively, for $24 \mathrm{~h}$. The experiment was performed for three times. All data were presented as mean \pm SEM. Compared with the control group, ${ }^{* *} P<0.01,{ }^{* *} P<0.001$ 

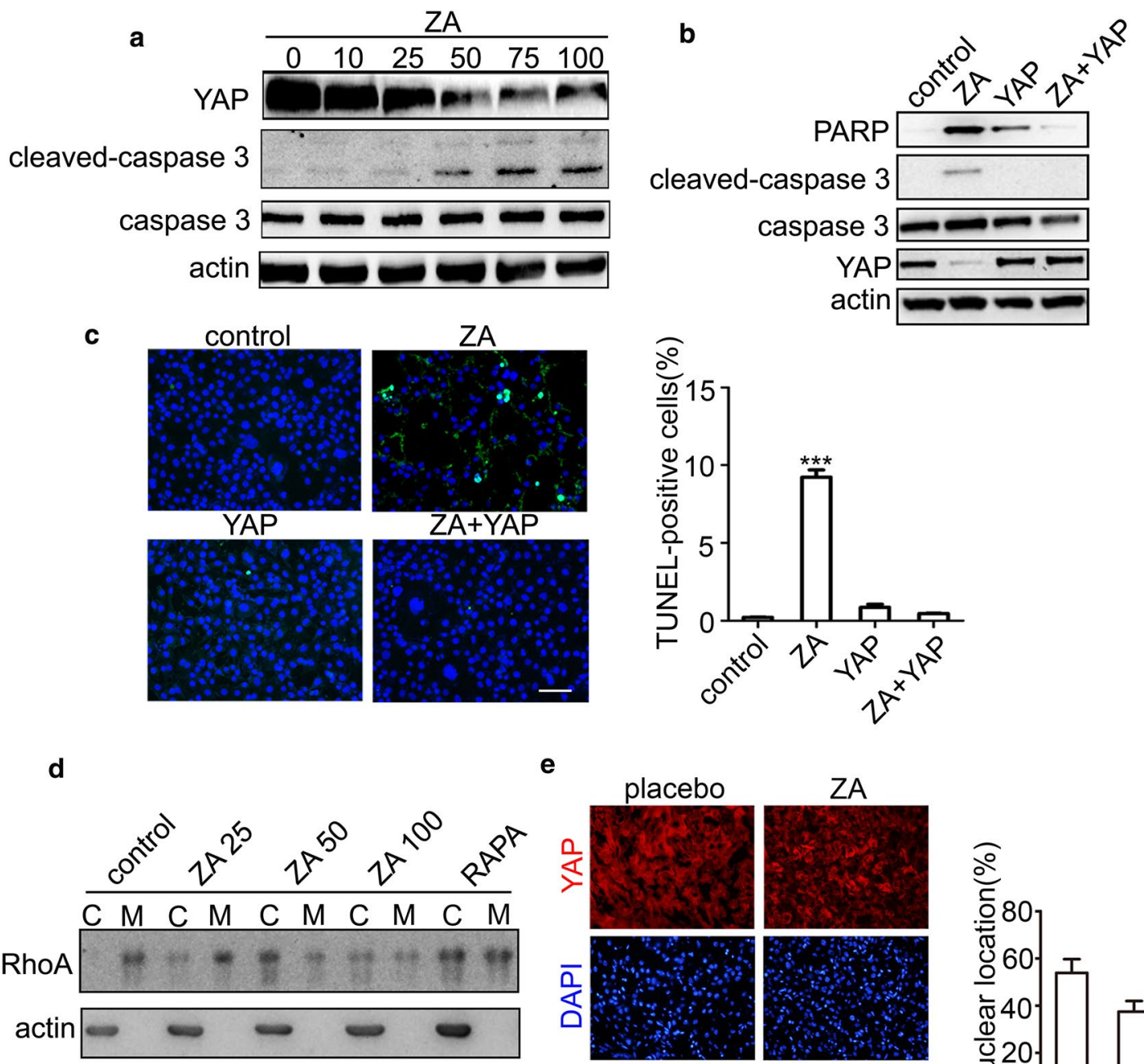

e

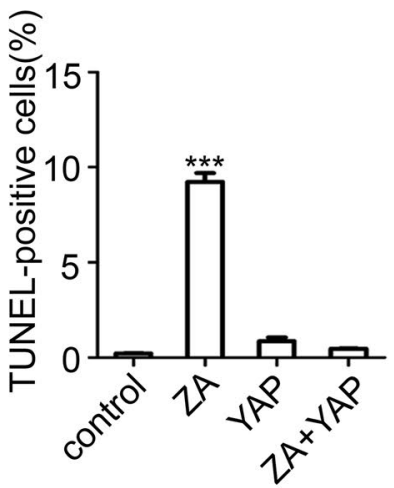

placebo
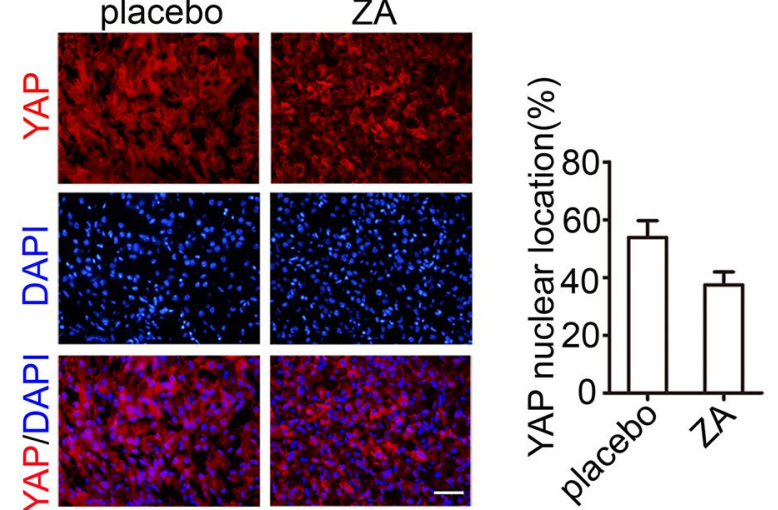

f
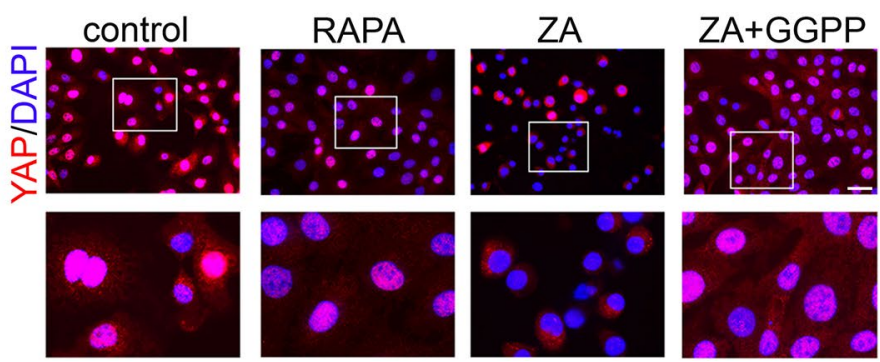

Fig. 3 ZA induced apoptosis by inhibiting YAP. a Yap, caspase-3, and cleaved caspase-3 levels were determined by the Western blot analysis, after the treatment of $0,10,25,50,75$, and $100 \mu \mathrm{M} Z \mathrm{ZA}$, respectively. $\mathbf{b}$ PARP and cleaved-caspase-3 levels were detected by the Western blot analysis after the treatment with ZA, YAP over-expression plasmid, and ZA and YAP over-expression plasmid, respectively. c Apoptosis was determined by TUNEL assay. The green dots represented apoptotic cells, and DAPI (blue) indicated cell nuclei. Scale bar, $100 \mu \mathrm{m}$. $\mathbf{d}$ Immunoblotting detection of RhoA localization at the plasma membrane of TSC2-null cells treated with control, ZA (25, 50, and $100 \mu \mathrm{M})$, and rapamycin (20 nM) for $24 \mathrm{~h}$ with serum. Scale bar, 50 rm. e Immunofluorescence analysis of Yap localization of TSC2-null cells in subcutaneous mouse tumors. Percentages of Yap-nuclear localized cells were calculated in different tumor lesions. Scale bar, $200 \mu \mathrm{m}$. f Yap translocation was determined by immunofluorescence after treated with rapamycin, ZA, and combination of ZA and GGPP. Scale bar, $50 \mu \mathrm{m}$. The experiment was performed for three times. All data were presented as mean \pm SEM. Compared with the control group, ${ }^{* * *} P<0.001$ 
a

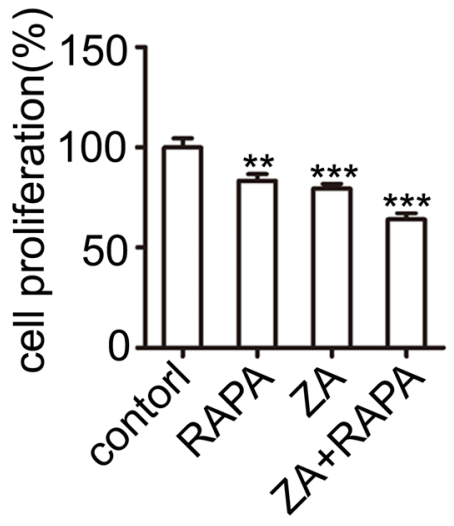

b

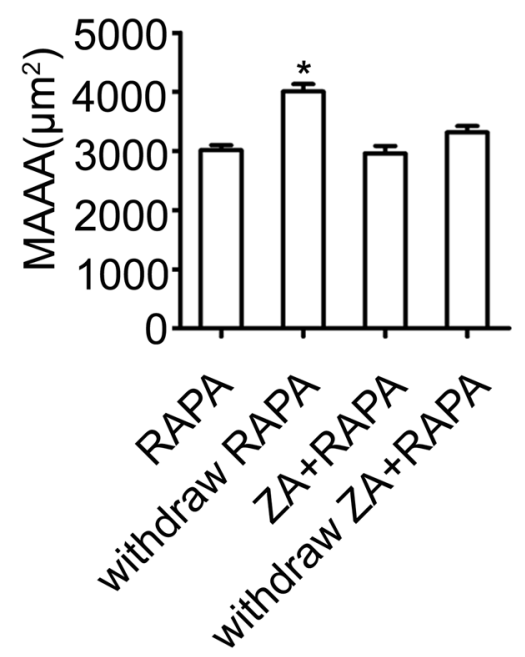

C

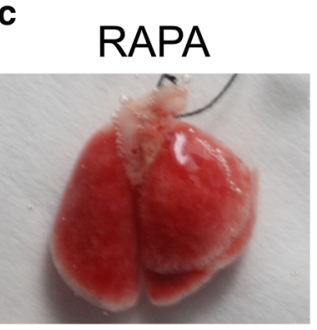

withdraw RAPA
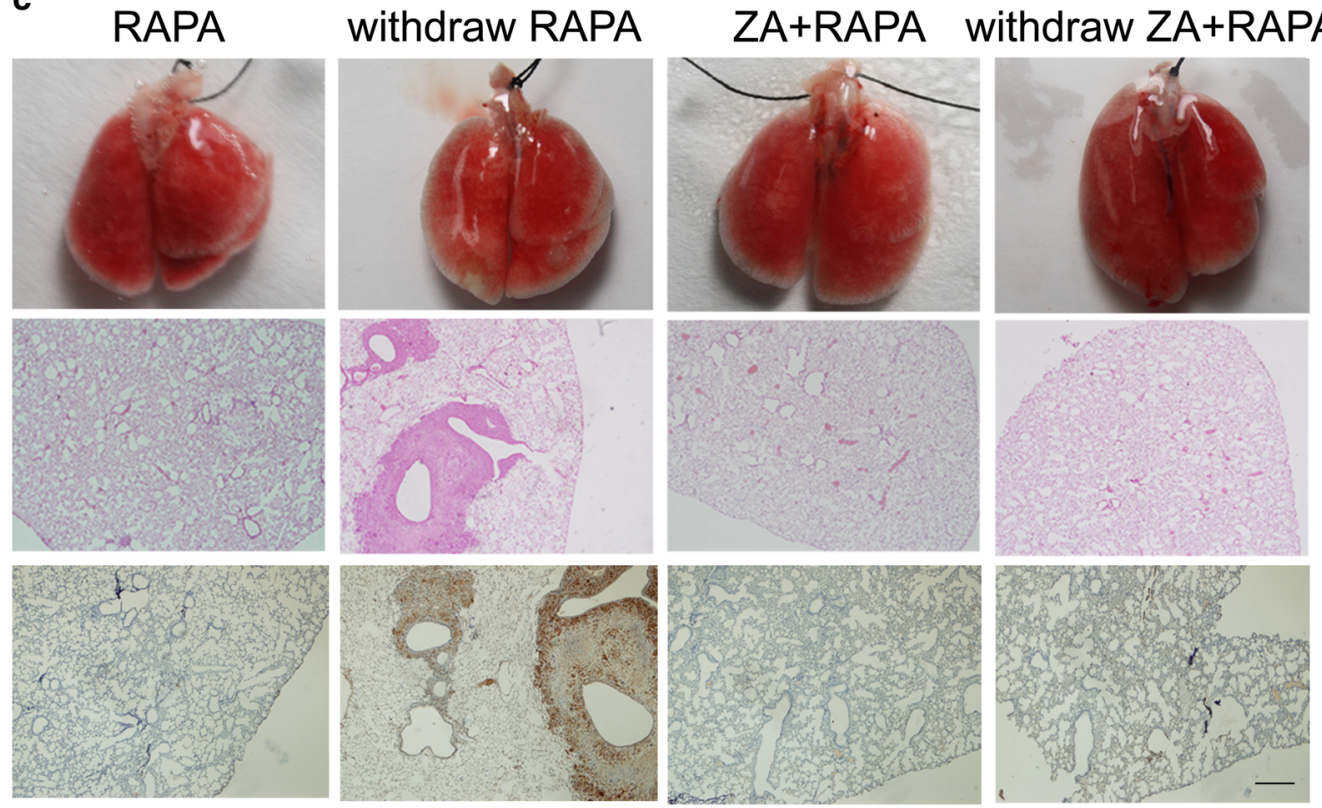

Fig. 4 Combination of ZA and rapamycin inhibits tumor recurrence after drug withdrawal. a Cell viability was determined by MTT after treated with rapamycin, ZA, and combination of ZA and rapamycin, respectively, for $24 \mathrm{~h}$. $\mathbf{b}$ Lung lesion was determined in the MAAA in LAM mouse model. c H\&E analysis of lung lesions and p-S6 staining of lung sections after injection of TSC2-null cells involving rapamycin (RAPA), the combination of RAPA and ZA-injected, and withdrawal therapy, after 30 day $(n=6)$. Scar bar, $500 \mu \mathrm{m}$. The experiment was performed for three times. All data were presented as mean \pm SEM. Compared with the control group, ${ }^{*} P<0.05$, ${ }^{* *} P<0.01$, ${ }^{* *} P<0.001$

combination of ZA and rapamycin could strongly inhibit the tumor progress and prevent the tumor recurrence in the LAM mouse models.

\section{Discussion}

Rapamycin is the only drug approved by FDA in the US and Japan for the clinical treatment of LAM. Lacking effective single therapeutic drugs for LAM makes the combination drug regimens necessary, such as the rapamycin in combination with statins and autophagy inhibitors. Currently, sirolimus (rapamycin) and hydroxychloroquine (autophagy inhibitor) have reached the clinical phase I [13]. The mevalonate pathway metabolites are essential for cancer cell survival and growth. ZA and simvastatin are inhibitors of the mevalonate pathway, with different target sites. The combination of rapamycin and simvastatin exhibits efficacy in LAM mouse models [25]. However, tumor recurrence after rapamycin treatment is a common problem in the clinical treatment of LAM, and there has been no good solution. In this study, 
for the first time, we validated that the combination of $\mathrm{ZA}$ and rapamycin, targeting on the mTOR and mevalonate pathways, could eliminate the tumors and inhibit tumor recurrence in the LAM mouse models.

Among small GTPases, RhoA and Rheb are essential parts of mTORC1 and mTORC2, respectively [27]. In addition, Ras activates the PI3K/AKT pathway, associated with the mTOR pathway [28]. ZA is an inhibitor of the mevalonate pathway, which inhibits the prenylation of small GTPases (i.e., the Ras, Rho, and Rac) [29]. We inferred that ZA could be an effective drug for apoptosis of TSC2-null cells. Indeed, our results showed that ZA not only inhibited the proliferation (just like rapamycin), but also promoted the apoptosis, of TSC2-null cells. More importantly, the combination of ZA and rapamycin absolutely eliminated the tumor, and no tumor recurrence occurred after drug withdrawal in the LAM mouse models. All these data suggests that the combination treatment of bisphosphonate and rapamycin provides new treatment method for LAM.

How TSC2 mutation leads to abnormal proliferation of LAM cells has not yet been fully elucidated. Some researches demonstrated that the excessive proliferation of TSC2-null cells is due to the accumulation of Yap in the nucleus [30,31]. The nuclear translocation of Yap is regulated by the RhoA protein, which requires the modification of GGPP [32]. We speculate that ZA might inhibit the Yap activity by blocking GGPP. The expression of Yap after ZA treatment was examined. Our results showed that, as the increase of ZA concentrations, the expression levels of Yap were gradually reduced in the TSC2-null cells. Moreover, the YAP over-expression could reverse the apoptosis induced by ZA. These results demonstrate that ZA could induce apoptosis of TSC2null cells by inhibiting Yap. Our results also showed that the RhoA protein bound on the cell membrane was significantly decreased, along with the increase concentrations of ZA. Moreover, the autophagic process was also triggered by the $\mathrm{ZA}$ treatment.

The limitation of our study is that the effects of ZA in patient LAM cells have not been verified. In recent years, high medical research and development costs limit the development of many orphan drugs, but the development of new clinical drugs can shorten the development cycle and save the development costs. ZA has been used in the clinical treatment of osteoporosis, with validated safety [33].

\section{Conclusion}

Our results showed that ZA affected the nuclear degradation of Yap and caused apoptosis through the protein prenylation. Our research provides new multidrug combination treatment of bisphosphonate and mTOR inhibitors for the mevalonate and mTOR pathways. These findings suggest that ZA might represent a promising pharmaceutical therapeutic drug for LAM.

\section{Supplementary information}

Supplementary information accompanies this paper at https://doi. org/10.1186/s12935-020-1131-4.

Additional file 1: Table S1. Antibodies and chemicals.

Additional file 2: Fig. S1. ZA induces autophagy in TSC2-null cells. (A) Phosphorylated $\mathrm{S} 6$ and Yap expression detected by immunoblotting analysis in TSC2-null cells treated with ZA $(25,50$, and $100 \mu \mathrm{M}$, respectively) for $24 \mathrm{~h}$. (B) LC3 expression was detected by immunoblotting analysis in TSC2-null cells treated with ZA $(25,50,75$, and $100 \mu \mathrm{M}$, respectively) for $24 \mathrm{~h}$. (C) Localization of LC3 and LAMP1 was analyzed by immunofluorescence in TSC2-null cells treated with ZA ( 25 and $50 \mu \mathrm{M}$, respectively) alone and RAPA (20 nM) for $24 \mathrm{~h}$. (D) Localization of LC3 and LAMP1 was analyzed by immunofluorescence in TSC2-null cells treated with ZA (50 $\mu \mathrm{M})$, RAPA $(20 \mathrm{nM})$, and combination of ZA $(50 \mu \mathrm{M})$ and GGPP $(20 \mu \mathrm{M})$ for $24 \mathrm{~h}$. The experiment was performed for three times. All data were presented as mean \pm SEM. Compared with the placebo, ${ }^{*} P<0.05$.

Additional file 3: Fig. S2. LAM mouse model establishment. (A) Analysis of lung lesions and immunohistochemical staining of $\mathrm{p}-\mathrm{S} 6$ in LAM mouse models after tail vein injection of TSC2-null cells for 30 day. Scar bar, 50, 200, and $500 \mu \mathrm{m}$, respectively. (B) H\&E staining of liver, heart, and kidney after tail vein injection of TSC2-null cells for 30 day. Scar bar, $500 \mu \mathrm{m}$. (C) Analysis of MAAA of lungs after tail vein injection of TSC2-cells for 30 day.

Additional file 4: Fig. S3. ZA induce autophagy leading to Yap protein degradation in TSC2-null cells. (A) Cell viability was determined by MTT after treated with rapamycin, $Z A$, and combination of $Z A$ and $C Q$, respectively, for $24 \mathrm{~h}$. (B) Yap translocation was determined by immunofluorescence after treated with rapamycin, $Z A$, and combination of $Z A$ and CQ, for $24 \mathrm{~h}$. Scale bar, 50um. (C) Quantitative real-time PCR analysis of YAP mRNA expression after ZA treatment with 25uM, 50uM, 100uM, for $24 \mathrm{~h}$.

The experiment was performed for three times. All data were presented as mean \pm SEM. Compared with the placebo, ${ }^{*} \mathrm{P}<0.05$.

\section{Abbreviations}

ZA: Zoledronic acid; LAM: Lymphangioleiomyomatosis; TSC: Tuberous sclerosis complex.

\section{Acknowledgements}

The authors would like to thank Translational Medicine Core Facilities, Medical School of Nanjing University.

\section{Authors' contributions}

DZ, wrote the manuscript and analyzed the data. JW, analyzed the data of immunofluorescence pictures and revised the manuscript. $Y Z$, contributed to data analysis of signaling pathway. WS, performed immunoblotting experiments. QC, performed qRT-PCR. XS, in vivo antitumor experiment. XY, performed the immunofluorescence experiments. JY, provided the compounds. $J G$, performed cell culture. MJ, provided technical assistance about the mouse model. CL, analyzed the manuscript for important intellectual content. XC, analyzed the data of mouse model. $Y Z$, designed the experiment and revised manuscript. BX, designed the experiment. All authors read and approved the final manuscript.

\section{Funding}

This work was supported by National Natural Science Foundation of China (NSFC) $(31371373,31771572,31100448$, and 31601153), National Natural Science Foundation of Jiangsu Province (BK20151395, BK20160619), and the Open Fund of State Key Laboratory of Natural Medicines (No. SKLNMKF201811), the Fundamental Research Funds for the Central Universities $(14380345,14380269,14380343)$, six talent peaks project in Jiangsu Province (yy-014), Priority Academic Program Development of Jiangsu Higher Education Institutions (PADA), China Postdoctoral Science Foundation (2016M601778). 


\section{Availability of data and materials}

Not applicable.

\section{Ethics approval and consent to participate}

The ethics committee of Nanjing University Medical School has approved this study.

\section{Consent for publication}

All authors read and approved the final manuscript and consent for publication.

\section{Competing interests}

All authors declare that they have no competing interests.

\section{Author details}

${ }^{1}$ Core Laboratory, Sir Run Run Hospital, Nanjing Medical University, Nanjing 211166, Jiangsu, China. ${ }^{2}$ State Key Laboratory of Pharmaceutical Biotechnology, Jiangsu Key Laboratory of Molecular Medicine and School of Medicine, Nanjing University, Nanjing 210093, Jiangsu, China. ${ }^{3}$ Collaborative Innovation Center of Sustainable Forestry in Southern China, College of Forestry, Nanjing Forestry University, Nanjing 210037, Jiangsu, China. ${ }^{4}$ Department of Hepatobiliary Surgery, The Affiliated Drum Tower Hospital of Nanjing University Medical School, Nanjing 210008, Jiangsu, China. ${ }^{5}$ Affiliated Hospital of Integrated Traditional Chinese and Western Medicine, Nanjing University of Chinese Medicine, Nanjing 210028, Jiangsu, China. ${ }^{6}$ Pulmonary Department, Zhongshan Hospital, Fudan University, Shanghai 200032, China. 7 Jiangsu Key Laboratory of Molecular Medicine and School of Medicine, Nanjing University, Nanjing 210093, Jiangsu, China. ${ }^{8}$ State Key Laboratory of Natural Medicines, China Pharmaceutical University, Nanjing 210009, Jiangsu, China. ${ }^{9}$ Respiratory Department, Sir Run Run Hospital, Nanjing Medical University, Nanjing 211166, Jiangsu, China.

Received: 4 September 2019 Accepted: 29 January 2020

Published online: 10 February 2020

\section{References}

1. Atochina-Vasserman EN, Guo C-J, Abramova E, Golden TN, Sims M, James ML, Beers MF, Gow AJ, Krymskaya VP. Surfactant dysfunction and lung inflammation in the female mouse model of lymphangioleiomyomatosis. Am J Respir Cell Mol Biol. 2015:53(1):96-104.

2. Baldi BG, Freitas CSG, Araujo MS, Dias OM, Pereira DAS, Pimenta SP, Kairalla RA, Carvalho CRR. Clinical course and characterisation of lymphangioleiomyomatosis in a Brazilian reference centre. Sarcoidosis Vasc Diffuse Lung Dis. 2014;31(2):129-35.

3. Costello LC, Hartman TE, Ryu JH. High frequency of pulmonary lymphangioleiomyomatosis in women with tuberous sclerosis complex. Mayo clinic proceedings. New York: Elsevier; 2000. p. 591-4.

4. Farach LS, Pearson DA, Woodhouse JP, Schraw JM, Sahin M, Krueger DA, Wu JY, Bebin EM, Lupo PJ, Au KS. Tuberous sclerosis complex genotypes and developmental phenotype. Pediatr Neurol. 2019;96:58-63.

5. Atochina-Vasserman EN, Abramova E, James ML, Rue R, Liu AY, Ersumo NT, Guo C-J, Gow AJ, Krymskaya VP. Pharmacological targeting of VEGFR signaling with axitinib inhibits Tsc2-null lesion growth in the mouse model of lymphangioleiomyomatosis. Am J Physiol Lung Cell Mol Physiol. 2015:309(12):L1447-54

6. Glasgow CG, Pacheco-Rodriguez G, Steagall WK, Haughey ME, JulienWilliams PA, Stylianou MP, Gochuico BR, Moss J. CA-125 in disease progression and treatment of lymphangioleiomyomatosis. Chest. 2018;153(2):339-48.

7. Meraj R, Wikenheiser-Brokamp KA, Young LR, McCormack FX. Lymphangioleiomyomatosis: new concepts in pathogenesis, diagnosis, and treatment. Seminars in respiratory and critical care medicine. New York: Thieme Medical Publishers; 2012. p. 486-97.

8. Muzykewicz DA, Sharma A, Muse V, Numis AL, Rajagopal J, Thiele EA. TSC1 and TSC2 mutations in patients with lymphangioleiomyomatosis and tuberous sclerosis complex. J Med Genet. 2009:46(7):465-8.

9. Carsillo T, Astrinidis A, Henske EP. Mutations in the tuberous sclerosis complex gene TSC2 are a cause of sporadic pulmonary lymphangioleiomyomatosis. Proc Natl Acad Sci. 2000;97(11):6085-90.
10. Inoki K, Li Y, Xu T, Guan K-L. Rheb GTPase is a direct target of TSC2 GAP activity and regulates mTOR signaling. Genes Dev. 2003;17(15):1829-34.

11. Tyryshkin A, Bhattacharya A, Eissa NT. SRC kinase is a novel therapeutic target in lymphangioleiomyomatosis. Cancer Res. 2014;74(7):1996-2005.

12. Slomovitz BM, Coleman RL. The PI3K/AKT/mTOR pathway as a therapeutic target in endometrial cancer. Clin Cancer Res. 2012;18(21):5856-64.

13. El-Chemaly S, Taveira-Dasilva A, Goldberg HJ, Peters E, Haughey M, Bienfang D, Jones AM, Julien-Williams P, Cui Y, Villalba JA. Sirolimus and autophagy inhibition in lymphangioleiomyomatosis: results of a phase I clinical trial. Chest. 2017;151(6):1302-10.

14. Davies DM, Johnson SR, Tattersfield AE, Kingswood JC, Cox JA, McCartney DL, Doyle T, Elmslie F, Saggar A, de Vries PJ. Sirolimus therapy in tuberous sclerosis or sporadic lymphangioleiomyomatosis. N Engl J Med. 2008;358(2):200-3.

15. McCormack FX, Inoue Y, Moss J, Singer LG, Strange C, Nakata K, Barker AF, Chapman JT, Brantly ML, Stocks JM. Efficacy and safety of sirolimus in lymphangioleiomyomatosis. N Engl J Med. 2011;364(17):1595-606.

16. Taveira-DaSilva AM, Hathaway O, Stylianou M, Moss J. Changes in lung function and chylous effusions in patients with lymphangioleiomyomatosis treated with sirolimus. Ann Intern Med. 2011;154(12):797-805.

17. Feldman ME, Apsel B, Uotila A, Loewith R, Knight ZA, Ruggero D, Shokat KM. Active-site inhibitors of mTOR target rapamycin-resistant outputs of mTORC1 and mTORC2. PLoS Biol. 2009;7(2):e1000038.

18. Mullen PJ, Yu R, Longo J, Archer MC, Penn LZ. The interplay between cell signalling and the mevalonate pathway in cancer. Nat Rev Cancer. 2016;16(11):718.

19. Luckman SP, Hughes DE, Coxon FP, Russell RGG, Rogers MJ. Nitrogen-containing bisphosphonates inhibit the mevalonate pathway and prevent post-translational prenylation of GTP-binding proteins, including Ras. J Bone Miner Res. 1998;13(4):581-9.

20. Goldstein JL, Brown MS. Regulation of the mevalonate pathway. Nature 1990;343(6257):425.

21. Taveira-DaSilva AM, Jones AM, Julien-Williams PA, Stylianou M, Moss J. Retrospective review of combined sirolimus and simvastatin therapy in lymphangioleiomyomatosis. Chest. 2015;147(1):180-7.

22. Taveira-DaSilva AM, Jones AM, Julien-Williams P, Stylianou M, Moss J. Long-term effect of sirolimus on serum vascular endothelial growth factor D levels in patients with lymphangioleiomyomatosis. Chest. 2018;153(1):124-32

23. Sunaga T, Shimamoto K, Nakamura S, Takahashi N, Higashino M, Hozumi T, Matsui M, Nagatani A, Kokubu F, Kogo M. The association between fever and prognosis in lung cancer patients with bone metastases receiving zoledronic acid. Chemotherapy. 2017;62(6):327-33.

24. Kim YN, Choe SR, Cho KH, Kang J, Park CG, Lee HY. Resveratrol suppresses breast cancer cell invasion by inactivating a RhoA/YAP signaling axis. Exp Mol Med. 2017;49(2):e296-e296.

25. Goncharova EA, Goncharov DA, Fehrenbach M, Khavin I, Ducka B, Hino O, Colby TV, Merrilees MJ, Haczku A, Albelda SM. Prevention of alveolar destruction and airspace enlargement in a mouse model of pulmonary lymphangioleiomyomatosis (LAM). Sci Transl Med. 2012;4(154):154.

26. Bordier C. Phase separation of integral membrane proteins in Triton X-114 solution. J Biol Chem. 1981;256(4):1604-7.

27. Nguyen TP, Frank AR, Jewell JL. Amino acid and small GTPase regulation of mTORC1. Cell Log. 2017;7(4):e1378794.

28. Gulhati P, Bowen KA, Liu J, Stevens PD, Rychahou PG, Chen M, Lee EY, Weiss HL, O'Connor KL, Gao T. mTORC1 and mTORC2 regulate EMT, motility, and metastasis of colorectal cancer via RhoA and Rac1 signaling pathways. Cancer Res. 2011;71(9):3246-56.

29. Wood J, Bonjean K, Ruetz S, Bellahcène A, Devy L, Foidart JM, Castronovo $V$, Green JR. Novel antiangiogenic effects of the bisphosphonate compound zoledronic acid. J Pharmacol Exp Ther. 2002;302(3):1055-61.

30. Dill PE, Liang N, Pende M. New insights into the pathophysiology of the tuberous sclerosis complex: crosstalk of mTOR-and hippo-YAP pathways in cell growth. Rare Dis. 2015:3(1):e1016701.

31. Shen Y, Pena A, Kudryashova T, Goncharov D, Chang B, Mora A, Baust J, Chavez Barragan A, Ray A, Rode A. Stiffness-induced TSC2 deficiency promotes vascular smooth muscle remodeling and pulmonary hypertension Via Yap/mTOR axis. In: A97 station to station: unraveling the molecular pathogenesis of PAH. American Thoracic Society; 2019. p. A2399. 
32. Sorrentino G, Ruggeri N, Specchia V, Cordenonsi M, Mano M, Dupont S, Manfrin A, Ingallina E, Sommaggio R, Piazza S. Metabolic control of YAP and TAZ by the mevalonate pathway. Nat Cell Biol. 2014;16(4):357.

33. Kubista B, Trieb K, Sevelda F, Toma C, Arrich F, Heffeter P, Elbling L, Sutterlüty H, Scotlandi K, Kotz R. Anticancer effects of zoledronic acid against human osteosarcoma cells. J Orthop Res. 2006;24(6):1145-52.

\section{Publisher's Note}

Springer Nature remains neutral with regard to jurisdictional claims in published maps and institutional affiliations.
Ready to submit your research? Choose BMC and benefit from:

- fast, convenient online submission

- thorough peer review by experienced researchers in your field

- rapid publication on acceptance

- support for research data, including large and complex data types

- gold Open Access which fosters wider collaboration and increased citations

- maximum visibility for your research: over 100M website views per year

At BMC, research is always in progress.

Learn more biomedcentral.com/submissions 